\title{
Molecular analysis of genital HPV: results of a study made in the years 2005-2008
}

\author{
Maria Di Bello, Giuseppe Lenoci, Carmela Mazzone, Michele Direnzo \\ Laboratorio di Biologia Molecolare, Ospedale Generale Regionale “F. Miulli", Acquaviva delle Fonti (BA)
}

Key-words: Polymerase Chain Reaction, HPV-DNA test, Condylomi, Eso-endocervical swabs

Analisi molecolare di HPV genitale: risultati di uno studio effettuato negli anni 2005-2008

\section{SUMMARY}

Background: Human Papilloma virus (HPV) infection is the main cause of cervical cancer and cervical intraepithelial neoplasia (CIN) worldwide. Consequently, it would be useful to evaluate HPV testing to screen for cervical cancer. Recently several molecular biological tests able to detect different HPV types and to classified them into high and low-risk groups have been developed. In this study we examined HPV prevalence and genotype distribution in a group of 346 patients, some women undergoing cytological screening for prevention, others with abnormal cytological and/ or clinics.

Methods: HPV detection and genotyping were done using a polymerase chain reaction based assay with the use the HPVTyping kit (Nuclear Laser Medicine). The tests can detect and differentiate 14 types of human papilloma virus, classified them into low, medium and high risk. Results. During the period 2005-2008, a total of 346 samples were analysed. The results of this study show that in this series, HPV infection were detected in $43.35 \%$ with a prevalence of $83.3 \%$ in patients of $16-20$ years.

Over the years there weren't an increase of the infection. Among patients with condylomas, the genotype most frequently detected were HPV 6 (79\%) followed by HPV II (25.5\%) while among the eso-endocervical swabs were HPV 16 (34.5\%) and HPV 6 (26.1\%).

Conclusions: Our work shows how is high the incidence of HPV infection in people especially young, thus the importance of HPV molecular test for diagnosing the presence of high risk HPV oncogenes. This epidemiological study can better understand the incidence of HPV in local and identification of the virus strain that maintains the infection, in order to assume a growing importance in prevention programs.

\section{INTRODUZIONE}

L’infezione da Human Papilloma Virus (HPV), considerata ad altissima diffusione e sessualmente trasmessa, è responsabile, oltre all'insorgenza dei condilomi genitali, anche e soprattutto del cancro della cervice uterina e dei suoi precursori, come le neoplasie intraepiteliali cervicali (CIN) $(1,6,12)$.

Il papillomavirus è un virus appartenente alla famiglia dei Papovavirus. Il capside nudo è composto da 72 capsomeri a simmetria icosaedrica a diametro compreso tra i 45 e i $55 \mathrm{~nm}$. Contiene un DNA a doppia elica (8000 bp) organizzato in sequenze nucleotidiche (ORFs) distinte in: geni L (L1, L2) che codificano per proteine strutturali del capside virale e geni E (E1, E2, E4, E5, E6, E7) che codificano per proteine regolatrici la replicazione del DNA virale.

Questi virus hanno un tropismo selettivo nei confronti di cellule degli epiteli squamosi e delle mucose. Gli HPV che infettano l'epitelio cervico-vaginale si possono distinguere in: HPV a basso rischio e HPV ad alto rischio.

Ne sono stati identificati più di 150 tipi, dei quali circa 40 a tropismo mucoso possono provocare infezioni del tratto anogenitale. Alcuni (i tipi 16, 18, 45, 31, 33, 35, 39, 51, 52, 56, 59, 68, $73,82)$ sono associati ad un rischio più elevato di progressione verso lesioni maligne, rispetto ad altri (i tipi $6,11,42,43,44)$ che sono solitamente responsabili dei condilomi e delle LG-SIL (Low Grade Squamous Intraepithelial Lesions) $(13,14)$.

Una volta che il virus ha infettato le cellule epiteliali, ha un periodo di incubazione di circa 3 mesi, dopo di che il suo destino può avere diverse evoluzioni (2). Nella maggior parte dei casi (80-90\%) viene eliminato spontaneamente con la clearance del virus entro 12-24 mesi. Nel 10\% circa dei casi, il virus si integra nel genoma della cellula ospite, incrementando il rischio di progressione verso la neoplasia nell'arco di 10-15 anni, e nel 10-15\% dei casi, rimane in forma episomiale all'interno della cellula, inducendo attraverso la propria replicazione la proliferazione dell'epitelio squamoso, producendo forme vegetative come i condilomi.

L'azione oncogena del virus si manifesta attraverso il processo di inattivazione di alcune molecole regolatrici del ciclo cellulare che, in condizioni normali funzionano come freno inibitore per la proliferazione cellulare, queste proteine sono: p53 prodotto del gene oncosoppressore omonimo e p105 Rb, prodotto del gene del retinoblastoma; rispettivamente hanno un elevata affinità per E6, E7. Venendo meno l'azione di blocco mediata da p53 e pRb, il virus può replicarsi con l'ausilio degli enzimi cellulari e propagarsi attraverso la lisi della cellula ospite.

In questi ultimi anni, l'utilizzo di test molecolari per la ricerca di HPV ad alto rischio oncogeno ha trovato ampia diffusione come strumento diagnostico fondamentale per l'implementazione dell'esame citologico nella prevenzione del carcinoma del collo dell'utero e nel follow up di pazienti trattate per lesioni di alto grado $(9,11)$.

Le linee guida nazionali ed internazionali come GISCi (Gruppo Italiano Screening del Cervicocarcinoma) e ASCCP (American Society of Colposcopy and Cervical Pathology) sostengono ormai da tempo l'affiancamento del test HPV al tradizionale Pap Test nel procedimento diagnostico delle SIL. Lo scopo di questo lavoro è stato quello di:

a) studiare l'epidemiologia dell'infezione da HPV nella nostra realtà locale valutando la prevalenza dell'infezione nel gruppo di pazienti analizzati, suddivisi per sesso maschile e femminile, ed età;

b) valutare la tipizzazione genotipica;

c) osservare l'andamento dell'infezione nel corso degli anni (2005-2008).

\section{MATERIALI E METODI}

Il nostro studio è stato effettuato nel periodo 2005-2008, presso il Laboratorio di Biologia Molecolare del P.O. "F. Miulli” di Acquaviva delle Fonti (BA). L’indagine è stata condotta su pazienti pervenuti nel nostro laboratorio come ricoverati e ambulatoriali, i quali nella maggior parte dei casi erano soggetti sottoposti a screening citologico di prevenzione $(70 \%)$, altri presentavano già alterazioni citologiche e/o cliniche (30\%).

I campioni biologici pervenuti in laboratorio sono stati 346, tra cui tamponi eso-endocervicali, tamponi uretrali e condilomi, analizzati nell'arco di tempo considerato.

I tamponi pervenuti sono stati raccolti mediante brushing cervicale. Il campione prelevato è stato successivamente inseri-

\section{Corresponding author: Carmela Mazzone}

Laboratorio di Biologia Molecolare, Ospedale Gen. Reg. “F. Miulli”, Acquaviva delle Fonti (BA)

74017 Mottola (TA) - Via Salvatore del re, 4 - Tel.: 3496932742

E-mail: carmela.mazzone@yahoo.it 
to in un apposito flacone sterile contenente $0.5 \mathrm{ml}$ di soluzione di trasporto (una miscela di penicillina a $10000 \mathrm{U} / \mathrm{ml}$, streptomicina a $10 \mathrm{mg} / \mathrm{ml}$ e anfotericina a $25 \mathrm{mg} / \mathrm{ml}$ in fisiologica sterile) e conservato a $-20^{\circ} \mathrm{C}$ sino al momento dell'esecuzione della PCR (Nuclear Laser Medicine con Termociclatore Applied Biosystems).

Per la ricerca dell'HPV-DNA, è stato utilizzato il kit HPV Typing (Nuclear Laser Medicine srl) che consente l'estrazione del DNA, la rilevazione e tipizzazione dei vari tipi di papilloma-virus considerati a basso rischio $(6,11)$, a medio rischio $(33,35,52,56,39,58,59,66)$ e ad alto rischio (16, 18, 31, 45) di trasformazione neoplastica. Il metodo Multiplex PCR è basato sull'amplificazione simultanea di più sequenze nucleotidiche HPV specifiche.

Il test permette di rilevare la presenza e di differenziare 14 tipi di papilloma virus umani e viene eseguito in quattro reazioni per ogni campione. I papilloma-virus umani ricercati nel test hanno diversa lunghezza del frammento amplificato e questo permette la loro genotipizzazione.

Il DNA bersaglio del controllo interno è il DNA del gene $ß$-globinico GLOBINA che deve essere quindi presente in tutti i campioni.

Per ciascun campione sono state utilizzate quattro provette mix di primer:

PCR mix «16-35»: primers per i tipi virali 16, 31, 33, 35, nucleotidi, controllo interno.

PCR mix «18-59»: primers per i tipi virali 18, 39, 45, 59, nucleotidi, controllo interno.

PCR mix «52-66»: primers per i tipi virali 52, 56, 58, 66, nucleotidi, controllo interno.

PCR mix «6, 11»: primers per i tipi virali 6, 11, nucleotidi, controllo interno.

La rilevazione della presenza di amplificato specifico è stata eseguita mediante elettroforesi su gel d'agarosio al $2 \%$ contenente etidio bromuro. Per evidenziare le bande corrispondenti ai vari tipi virali è stato utilizzato un marcatore di pesi molecolari (50 bp DNA step leader) che evidenzia bande da 50 a 800 bp. Sono stati considerati negativi i campioni che contenevano solo la banda a 723 bp, corrispondente al controllo interno. I campioni positivi vengono classificati in base alla lunghezza del frammento amplificato di DNA.

\begin{tabular}{|c|c|}
\hline \multicolumn{2}{|c|}{ PCR-mix-I «6-II» } \\
\hline Genotipo & Lunghezza \\
\hline HPV 6 & $260 \mathrm{bp}$ \\
\hline HPV II & $425 \mathrm{bp}$ \\
\hline \multicolumn{2}{|c|}{ PCR-mix-2 «|6-35» } \\
\hline Genotipo & Lunghezza \\
\hline HPV I6 & $325 \mathrm{bp}$ \\
\hline HPV 3I & $520 \mathrm{bp}$ \\
\hline HPV 33 & $227 \mathrm{bp}$ \\
\hline HPV 35 & $280 \mathrm{bp}$ \\
\hline
\end{tabular}

\begin{tabular}{|c|c|}
\hline \multicolumn{2}{|c|}{ PCR-mix-3 «52-66» } \\
\hline Genotipo & Lunghezza \\
\hline HPV 52 & $360 \mathrm{bp}$ \\
\hline HPV 56 & 325 bp \\
\hline HPV 58 & $240 \mathrm{bp}$ \\
\hline HPV 66 & $304 \mathrm{bp}$ \\
\hline
\end{tabular}

\begin{tabular}{|c|c|}
\hline \multicolumn{2}{|c|}{ PCR-mix-4 «|8-59» } \\
\hline Genotipo & Lunghezza \\
\hline HPV 18 & $425 \mathrm{bp}$ \\
\hline HPV 39 & $340 \mathrm{bp}$ \\
\hline HPV 45 & 475 bp \\
\hline HPV 59 & $395 \mathrm{bp}$ \\
\hline
\end{tabular}

Nel caso dell'assenza della banda di 723 bp la seduta non è stata accettata in quanto l'assenza della banda del controllo interno indica la non idoneità del prelievo del campione clinico o un errore tecnico. La sensibilità e la specificità clini- che dichiarate del test sono rispettivamente del $96.0 \%$ e del $95.0 \%$. La sensibilità analitica del test è tale da consentire la rilevazione di circa 200 copie di HPVDNA/mL.

\section{RISULTATI}

Sono stati esaminati 346 campioni, di cui 51 (14.74\%) condilomi, 288 (83.23\%) tamponi eso-endocervicali, e 7 tamponi uretrali. La positività al test di ricerca di HPV-DNA eseguito tramite PCR, è stata del 43.35\% (150 campioni) così suddivisa: $28.6 \%$ (43 campioni) tra i condilomi, 70\% (105 campioni) tra i tamponi eso-endocervicali, e 1.3\% (2 campioni) tra i tamponi uretrali.

L'età media dei pazienti analizzati in questo studio è risultato di 29 anni, la distribuzione per fasce d'età dell'infezione è presentata nella Tabella 1.

L'intervallo di età che ha presentato il più elevato tasso di infezione (83.3\%) è risultato quello compreso tra 16-20 anni, mentre quello a titolo più basso (34.2\%) è stato quello riscontrato in pazienti con età maggiore di 35 anni.

Sul totale dei condilomi, pari a 51 (43 maschi e 8 femmine), la positività dell'HPV è stata del 70.5\% (36 campioni) tra i condilomi maschili e del 13.7\% (7 campioni) tra i condilomi femminili. Tra i tamponi la positività di HPV è stata del $36.3 \%$ pari a 107 campioni positivi (105 donne e 2 uomini relativi ai campioni uretrali) su un totale di 295.Nel periodo di studio considerato, è stato possibile notare un incremento delle richieste di ricerca del virus, mentre la percentuale di infezione da HPV è rimasta pressochè costante, in media del $41.25 \%$ (Figura I).

Tra i genotipi più frequentemente identificati mediante PCR a livello di condilomi vi sono quelli a "basso rischio" come HPV 6 (79\%) e HPV11 (25.5\%) seguiti in maniera più esigua da altri HPV mostrati in Tabella 2, mentre tra i tamponi esoendocervicali si presentano con un alta incidenza HPV 16 (34.5\%) considerato ad "alto rischio", seguito da HPV 6 (26,1\%) (Tabella 3).

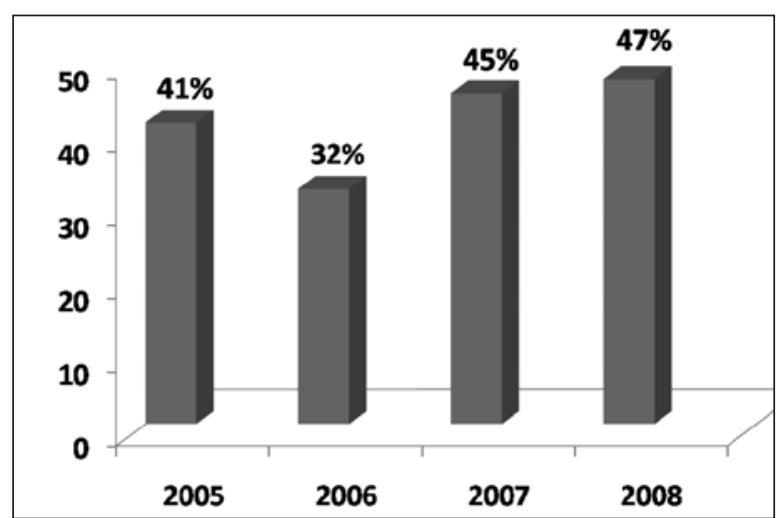

Figure I. Percentuale di positività per HPV riscontrata mediante metodo molecolare.

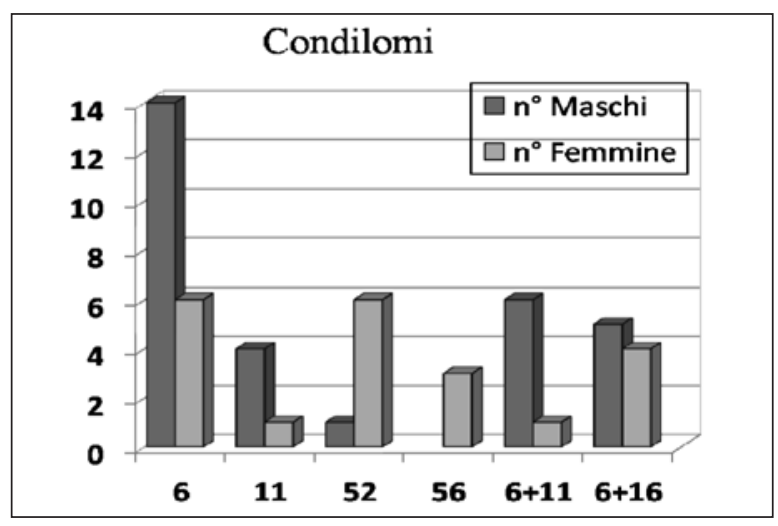

Figure II. Genotipo più frequentemente isolato nei condilomi in relazione al sesso. 
Tabella I. Infezione da HPV in rapporto all'età dei pazienti $(n=150)$

\begin{tabular}{lccccc}
\hline INTERVALLO ETA' & $\mathbf{1 6 - 2 0}$ & $\mathbf{2 I - 2 5}$ & $\mathbf{2 6 - 3 0}$ & $\mathbf{3 I - 3 5}$ & $\mathbf{3 5}$ \\
\hline Numero pazienti & 12 & 43 & 54 & 35 & I52 \\
\hline Numero positivi & 10 & 28 & 25 & 52 & \\
\hline Percentuale (\%) & 83.3 & 65.1 & 46.3 & $4 I .2$ & 34.2 \\
\hline
\end{tabular}

Tabella 2. Prevalenza dei genotipi HPV ad "alto e basso" rischio riscontrati nei campioni pervenuti come condilomi

\begin{tabular}{|c|c|c|c|c|c|c|c|c|c|c|c|c|c|}
\hline HPV & 6 & I I & 16 & 18 & 56 & 45 & 59 & 66 & 35 & 52 & 39 & 58 & 33 \\
\hline$\overline{n^{\circ} p z}$ & 34 & II & 8 & 5 & 3 & 2 & 2 & 2 & I & I & I & I & I \\
\hline$\%$ & 79 & 25.5 & 18.6 & 11.6 & 6.9 & 4.6 & 4.6 & 4.6 & 2.3 & 2.3 & 2.3 & 2.3 & 2.3 \\
\hline
\end{tabular}

Tabella 3. Prevalenza dei genotipi HPV ad "alto e basso" rischio riscontrati nei campioni pervenuti come tamponi eso-endocervicali

\begin{tabular}{|c|c|c|c|c|c|c|c|c|c|c|c|c|c|c|}
\hline HPV & 6 & 16 & 56 & 31 & 52 & 58 & 35 & I I & 45 & 59 & 18 & 33 & 39 & 66 \\
\hline$\overline{n^{\circ} p z}$ & 28 & 37 & 14 & II & 10 & 8 & 7 & 5 & 5 & 4 & 3 & 3 & 2 & 2 \\
\hline$\%$ & 26.1 & 34.5 & 13 & 10.3 & 9.3 & 7.5 & 6.5 & 4.7 & 4.7 & 3.7 & 2.8 & 2.8 & 1.8 & 1.8 \\
\hline
\end{tabular}

I dati mostrano che tra i virotipi ad alto rischio come il 16 e 18 riscontrati nei tamponi vi è una notevole differenza di frequenza, (rispettivamente del $34.5 \%$ e $2.8 \%$ ), lo stesso succede in quelli a basso rischio come il 6 e 11 identificati tra i condilomi (rispettivamente del 79\% e $25.5 \%$ ).

Sul totale di 150 pazienti HPV positivi, 46 (30.6\%) ha mostrato avere una coinfezione da HPV a basso ed alto rischio. È risultato che 23 (15.3\%) pazienti hanno contratto l'infezione da almeno un virotipo ad alto rischio. Limitata è risultata la contemporanea presenza dei virotipi 16-56 (5/150: 3.3\%), 616 (14/150: 9.3\%) e 6-11 (7/150: 4.6\%). Solamente in due casi (1.3\%) è stato identificato il virotipo 16-18.

Nelle pazienti infettate da HPV a basso rischio è stata osservata la presenza del virotipo 6 nel $42 \%$ dei casi, mentre nelle pazienti coinfettate da HPV a basso rischio e ad alto rischio il virotipo 6 è sempre stato riscontrato in associazione a 11, 16, 18 e/o 56, 58.

I virotipi più frequentemente isolati tra i condilomi in base al sesso, sono mostrati nella Figura II, mentre per i tamponi non è possibile fare una differenza di virotipo tra maschi e femmine, dal momento che i positivi maschi sono solo due relativi ai tamponi uretrali.

Non è stata presa in esame la relazione tra i risultati citologici e la ricerca molecolare del virus, dal momento che non erano disponibili i dati clinici.

\section{CONCLUSIONI}

L'HPV-DNA test è risultato positivo in 150 pazienti su un totale di 346 campioni, con una prevalenza del 43.35\%.

L'infezione da HPV ha una maggiore frequenza (83.3\%) all'esordio dell'attività sessuale (16-20 anni), dati in accordo con quanto riportato in letteratura (7).

Nel corso degli anni l'infezione da HPV non ha subito un incremento di incidenza, ciò nonostante la percentuale si mantiene comunque alta per via dei numerosi fattori di rischio, come del resto comunemente riportato in tutti i lavori che trattano la problematica di queste infezioni $(4,10,15$, 18). Per quanto riguarda la distribuzione dei virotipi di HPV nel gruppo di studio preso in esame, i risultati ottenuti (rilevazione del DNA virale tra i condilomi del tipo virale HPV 6 e HPV 11) sono in accordo con i risultati della casistica internazionale e nazionale $(5,16,17)$.

Tra i virotipi ad alto rischio prevale HPV 16 (34.5\%) nei tamponi eso-endocervicali mentre tra quelli a basso rischio emerge HPV 6 (79\%) nei condilomi. Inoltre il virotipo HPV 16 è risultato presente nel 14\% dei casi in associazione ad HPV 6, 56,18 , questo evidenzia la sua notevole caratteristica neoplastica nonché l'importanza di conoscere, in fase di screening, il tipo di virus da cui è sostenuta l'infezione per poterne valutare il grado di pericolosità futura ed impostare adeguati programmi di vaccinazione preventiva $(2,3,8,19)$.

\section{BIBLIOGRAFIA}

1. Bosch FX, Lorincz A, Munoz N, Meijer CJLM, Shah KV: The causal relation between human papillomavirus and cervical cancer. J Clin Pathol 2002; 55:244-265.

2. Capra G et al. HPV genotype prevalence in cytologically abnormal cervical samples from women living in south Italy. Virus Res 2008.; 133: 195-200.

3. Centurioni MG, Puppo A, Merlo DF, et al. Prevalence of human papillomavirus cervical infection in a Italian asymptomatic population. BMC Infectious Diseases 2006; 5: 77.

4. Clifford G, Franceschi S, Diaz M, Munoz N, Villa LL. Chapter 3: HPV typedistribution in women with and without cervical neoplatic diseases. Vaccine 2006; 24S3: S3/26-S3/34.

5. De Francesco MA, Gargiulo F, Schreiber C, Ciravolo G, Salinaro F, Manca N. Detection and genotyping of Human papillomavirus in cervical samples from Italian patients. J Med Virol 2005; 75: 588-92.

6. Dunne EF, Unger ER, Sternberg M, McQuillan G, Swan DC, Patel SS. Prevalence of HPV infection among females in the united states. JAMA 2007; 297: 813-19.

7. Frazer IH, Cox JT, Mayeaux EJ, Franco EL, et al. Advances in prevention of cervical cancer and other Human Papillomavirus-related diseases. Ped Infect Dis J 2006; 25: S65-81.

8. Franceschi S, Herrero R, Clifford G, Snijders PJF, Arslan A, Anh PTH et al. Variations in the age-specific curves of human papillomavirus prevalence in women worldwide. Int J Cancer 2006; 119: 2677-84.

9. Harper DM, Franco EL, Wheller CM, et al. Sustained efficacy up to 4.5 years of bivalent L1 virus-like particles against human papilloma virus type 16 and 18: follow-up from a randomised trial. Lancet 2006; 367: 1247-55.

10. Kitchener HC, Almonte M, Wheeler P, Desai M, Gilham C, Bailey A et al. HPV testing in routine cervical screening: cross sectional data from the ARTISTIC trial. Br J Cancer 2006; 95: 56-61.

11. Kahn JA, Lan D, Kahn RS. Sociodemographic factors associated with high-risk human papillomavirus infection. Obstet Gynecol. 2007; 110(3):713.

12. Ministero della Salute. Centro Nazionale per la Prevenzione ed il Controllo delle Malattie (CCM). Piano per lo screening del cancro del seno, della cervice uterina e del colon-retto. Legge n.138 del 26 Maggio 2004 (art.2 bis). Roma, 10 novembre 2004.

13. Munoz N. Human papillomavirus infection and cancer: the epidemiological evidence. J Clin Virol 2000; 19: 1-5.

14. Munoz N, Bosch FX, De Sanjosè S, et al. Epidemiologic classification of Human Papillomavirus types associated with cervical cancer. N Engl J Med 2003; 348: 518-27.

15. Nielson CM, Harris RB, Flores R, Abrahamsen M, Papenfuss MR, Dunne $\mathrm{EF}$, et al. Multiple-type human papillomavirus infection in male anogenital sites: prevalence and associated factors. Cancer Epidemiol Biomarkers Prev. 2009; 18(4):1077-83.

16. Nielsen A, Kjaer SK, Munk C, Iftner T. Type-specific HPV infection and multiple HPV types: prevalence and risk factor profile in nearly 12,000 younger and older Danish women. Sex Transm Dis. 2008; 35(3):276-82.

17. O’Keefe EJ, Gardner A, Currie MJ, Garland S, Tabrizi S, Bowden FJ. Prevalence of genital human papillomavirus DNA in a sample of senior school-aged women in the Australian Capital Territory. Sex Health. 2006; 3(2):91-4.

18. Ronco G, Ghisetti V, Segnan N, Snijders PFJ, Gillio-Tos A, Meijer CJLM et al. Prevalence of human papillomavirus infection in women in Turin, Italy. Eur J Cancer 2005; 41: 297-305.

19. Shikary T, Bernstein DI, Jin Y, Zimet GD, Rosenthal SL, Kahn JA. Epidemiology and risk factors for human papillomavirus infection in a diverse sample of low-income young women. J Clin Virol 2009; 4:12-19.

20. Ventura A, Carcheri M, Caci E, Caligiuri P, Capuzzo R, Chiossone I, et al. Valutazione del ruolo dell'HPV-DNA test nei programmi di prevenzione del cancro cervicale. Microbiologia Medica 2006; 21(4):349-56. 